This item was submitted to Loughborough's Research Repository by the author.

Items in Figshare are protected by copyright, with all rights reserved, unless otherwise indicated.

\title{
Highly porous magnetic Janus microparticles with asymmetric surface topology
}

PLEASE CITE THE PUBLISHED VERSION

https://doi.org/10.1021/acs.langmuir.0c02315

\section{PUBLISHER}

American Chemical Society

VERSION

AM (Accepted Manuscript)

\section{PUBLISHER STATEMENT}

This document is the Accepted Manuscript version of a Published Work that appeared in final form in Langmuir: the ACS journal of surfaces and colloids, copyright $\odot$ American Chemical Society after peer review and technical editing by the publisher. To access the final edited and published work see https://doi.org/10.1021/acs.langmuir.0c02315

\section{LICENCE}

CC BY-NC-ND 4.0

\section{REPOSITORY RECORD}

Alnuumani, Ruqaiya, SK Smoukov, Guido Bolognesi, and Goran Vladisavljevic. 2020. "Highly Porous Magnetic Janus Microparticles with Asymmetric Surface Topology". Loughborough University. https://hdl.handle.net/2134/13047953.v1. 


\section{Highly porous magnetic Janus microparticles with asymmetric surface topology}

Ruqaiya Al Nuumani, ${ }^{+}$Stoyan K. Smoukov, ${ }^{\$}$ Guido Bolognesi, ${ }^{+}$Goran T. Vladisavljević ${ }^{+*}$

${ }^{+}$Department of Chemical Engineering, Loughborough University, Loughborough, LE11 3TU, United Kingdom.

${ }^{\$}$ School of Engineering and Materials Science, Queen Mary University of London, London, E1 4NS, United Kingdom.

*Corresponding author (fax: +44 1509223923, email address: g.vladisavljevic@lboro.ac.uk)

\section{Graphical Abstract}

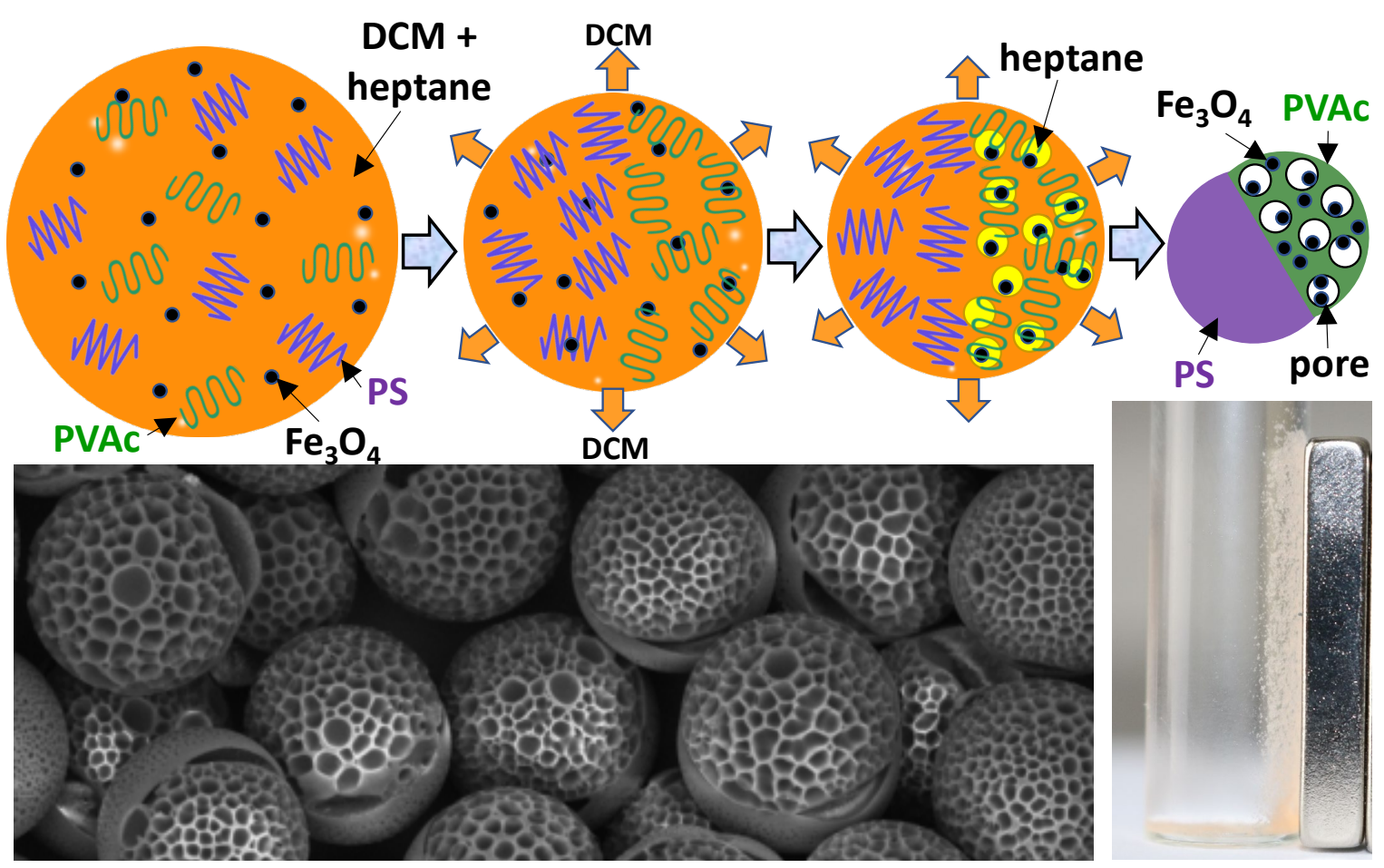


ABSTRACT. Monodispersed magnetic Janus particles composed of a porous polystyrene portion and a nonporous poly(vinyl acetate) portion with embedded oleic acid-coated magnetic nanoparticles were generated using microfluidic emulsification followed by two distinct phase separation events triggered by solvent evaporation. The template droplets were composed of 2 wt $\%$ polystyrene, $2 \mathrm{wt} \%$ poly(vinyl acetate) and 0.5-2 wt $\% n$-heptane-based magnetic fluid dissolved in dichloromethane (DCM). The porosity of polystyrene compartments was the result of phase separation between a non-volatile non-solvent ( $n$-heptane) and a volatile solvent (DCM) within polystyrene-rich phase. The focused ion beam cross-sectioning and SEM imaging revealed high surface porosity of polystyrene compartments with negligible porosity of poly(vinyl acetate) parts, which can be exploited to increase the wettability contrast between the two polymers and enhance bubble generation in bubble-driven micromotors. The porosity of the polystyrene portion was controlled by varying the fraction of $n$-heptane in the dispersed phase. The particle composition was confirmed by scanning electron microscopy-energy dispersive X-ray spectroscopy, Fourier transform infrared spectroscopy, and differential scanning calorimetry. The fabricated particles were successfully magnetised when subjected to an external magnetic field, which led to their aggregation into regular 2D assemblies. The particle clusters composed of 2-4 individual particles could be rotated with a rotating magnetic field. Microfluidic generation of highly porous Janus particles with compositional, topological, and magnetic asymmetry provides a cost-effective, easy-to-implement yet highly robust and versatile strategy for the manufacturing of multifunctional smart particles.

Keywords: Magnetic particles; Microfluidics; Phase separation; Porous polymer particles; Solvent evaporation method; material anisotropy; Janus particles.

\section{INTRODUCTION}

Janus particles (JPs) are particles with dissimilar chemical composition and bulk or surface properties on two opposite sides of the same particle leading to their anisotropic characteristics ${ }^{1-2}$. Due to the ability of JPs to combine diverse and often synergistic or incompatible functionalities in one structural entity, they can be used in a variety of sophisticated applications, e.g. as bicolored 
beads for electronic papers, ${ }^{3-5}$ dual imaging probes, ${ }^{6-7}$ drug delivery carriers, ${ }^{8-10}$ rheological probes, ${ }^{11}$ self-propelled micromotors, ${ }^{12}$ biphasic catalysts, ${ }^{13-15}$ and interface stabilisers. ${ }^{16-18}$

JPs can serve as efficient Pickering stabilisers for emulsions and foams as they combine the ability of solid particles to be adsorbed at fluid interfaces with the amphiphilicity of surfactants. ${ }^{16,19}$ High interfacial activity of JPs due to wettability contrast between their distinct poles can be combined with their ability to self-assemble into highly ordered suprastructures and used to create active functional interfaces with controlled chemical and topographical heterogeneity. ${ }^{20} \mathrm{JPs}$ can exhibit bubble-driven autonomous motion by running a gas-generating enzymatic reaction on one side of the particle. ${ }^{12}$ Furthermore, JPs can be used for simultaneous encapsulation of incompatible components within the same particle, ${ }^{21-23}$ which offers a promising strategy for enhanced efficacy of combination chemotherapy. ${ }^{24}$ Encapsulated drugs can be released at distinct rates from the two compartments if they are made of polymers with different biodegradation properties.

Magnetic JPs have attracted a lot of interest because they can be remotely manipulated, which allows their controlled translational and rotational motion and orientation. ${ }^{25-27}$ Magnetic JPs can provide targeted therapy and imaging and increased kinetics and sensitivity of analysis based on the use of external magnetic field in particle manipulation. ${ }^{7}$ The ability of magnetic particles to be torqued by an external magnetic field make them applicable as micro-stirrers, microrheological probes, and micro-magnetometers. ${ }^{28}$ Furthermore, they can easily be separated from a suspension by an externally imposed magnetic field and reused.

JPs have been fabricated using a variety of different methods including masking and asymmetric surface modification, ${ }^{29}$ self-assembly, ${ }^{30}$ seeded emulsion polymerization, ${ }^{31}$ reaction- or evaporation-induced internal phase separation, ${ }^{32-33}$ and microfluidics. ${ }^{34-36,9}$ Microfluidics remains a powerful technique for the fabrication of JPs due to its simplicity, low consumption of chemicals, reproducibility, and a high ability of controlling the shape, size and morphology of droplets. ${ }^{37}$ The two most common microfluidic methods for the fabrication of JPs are photopolymerisation of Janus droplets ${ }^{36}$ and solvent evaporation. ${ }^{33}$ In the former case, two co-flowing streams of different composition (each containing a photocurable monomer and photoinitiator) meet together in a $\mathrm{Y}$ junction to form Janus droplets, which are then polymerised after exposure to UV light in a 
downstream channel. ${ }^{38-41}$ In the solvent evaporation method, single phase droplets containing two polymers dissolved in a volatile solvent are hardened via solvent evaporation and the bifacial morphology is formed after phase separation of polymers. ${ }^{42-43}$

In this paper, we report a novel microfluidic strategy for fabrication of polymeric Janus particles with compositional, topological, and magnetic asymmetry, based on a single-step emulsification and solvent evaporation process with two distinct phase separation phenomena within the template droplets. Typically, polymeric Janus particles are formed by polymer-polymer phase separation in a ternary system containing two polymers and a solvent and such particles have a uniform topology across the entire surface. Here, we have implemented solvent-nonsolvent phase separation after polymer-polymer phase separation in a quinary system composed of two polymers (polystyrene, and poly(vinyl acetate)), a volatile good solvent (DCM), a bad solvent ( $n$-heptane), and oleic acid coated magnetic nanoparticles. Due to high polarity difference between the two polymers and strong preference of $n$-heptane to migrate into polystyrene phase, the porosity difference between the two polymer portions was much more pronounced than in previous studies ${ }^{33,44-45}$ with extremely high surface porosity of polystyrene parts.

\section{MATERIALS AND METHODS}

\section{Materials}

Polystyrene (PS, $\mathrm{M}_{\mathrm{W}} \sim 192,000 \mathrm{~g} / \mathrm{mol}$ ) and poly(vinyl acetate) (PVAc, $\mathrm{M}_{\mathrm{W}} \sim 100,000 \mathrm{~g} / \mathrm{mol}$ by GPC) obtained from Sigma-Aldrich (UK) were used as the main constituents of the Janus particles. Dichloromethane (DCM, HPLC grade, Fischer Scientific, UK) was used as a good solvent for both polymers. The magnetic fluid (oleic acid-stabilised iron oxide nanoparticles dispersed in $n$ heptane) was obtained from Sigma-Aldrich with a solid phase content of 0.8-1.4 wt\% and a Zaverage value of $\sim 33 \mathrm{~nm}$. Nile Red (9-diethylamino-5-benzo[a]phenoxazinone, Sigma-Aldrich) was added to the dispersed phase in trace amounts as a hydrophobic fluorescent dye to visualise the hydrophobic regions of JPs. Poly(vinyl alcohol) (PVA, $\mathrm{M}_{\mathrm{W}}=13,000-23,000 \mathrm{~g} / \mathrm{mol}, 87-89 \%$ hydrolysed, Sigma-Aldrich) was used as a hydrophilic stabiliser in the continuous phase. Glycerol (Fisher Scientific) was used to adjust the viscosity of the continuous phase. The reverse osmosis water was supplied using a Milli-Q apparatus. 


\section{Microfluidic set-up and procedure}

Monodispersed oil-in-water $(\mathrm{O} / \mathrm{W})$ emulsion droplets were generated in a flow-focusing glass capillary device shown in Fig. 1. The device was fabricated according to the procedure explained elsewhere. ${ }^{46-48}$ The dispersed and continuous phase liquids were supplied to the device using separate Harvard Apparatus 11 Elite syringe pumps via Teflon (PTFE) and polyethylene (PE) medical tubing, respectively. The dispersed phase was delivered at $3 \mathrm{ml} / \mathrm{h}$ from the left-hand side of the device through a square borosilicate capillary (I.D. $=1.05 \pm 0.1 \mathrm{~mm}$, AIT Glass, Rockaway, USA). The continuous phase was delivered at $25 \mathrm{ml} / \mathrm{h}$ from the opposite side of the same capillary. The droplets were generated inside a round borosilicate capillary (I.D. $=0.58 \mathrm{~mm}$, O.D. $=1.00$ mm, Intracel, UK) with a tapered tip shaped using a P-97 micropipette puller (Sutter Instrument Co., UK). Inverted biological microscope (XDS-3, GT-Vision, UK) and Phantom V9.0 high-speed camera interfaced to a PC computer were used to control the droplet generation process in real time.

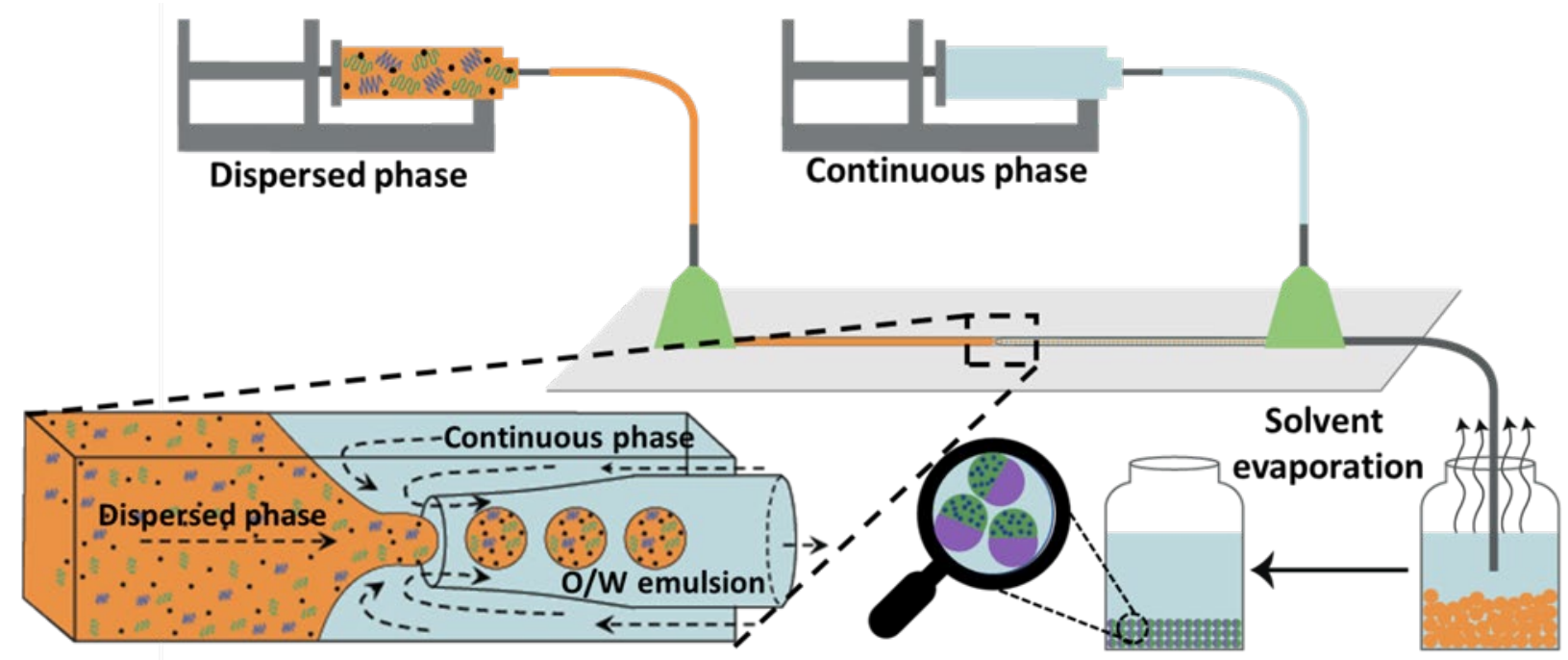

Fig 1. Schematic illustration of the experimental set-up used in this work. For Janus particles, the dispersed phase was $2 \mathrm{wt} \%$ PS $+2 \mathrm{wt} \%$ PVAc $+0.5-2 \mathrm{wt} \%$ magnetic fluid in DCM. For PS magnetic particles, the dispersed phase was $4 \mathrm{wt} \% \mathrm{PS}+0.5-4 \mathrm{wt} \%$ magnetic fluid in DCM. The continuous phase was $2 \mathrm{wt} \% \mathrm{PVA}+30 \mathrm{wt} \%$ glycerol in milli-Q water. 
Magnetic Janus particles were prepared from the dispersed phase containing $2 \mathrm{wt} \%$ PS, $2 \mathrm{wt} \%$ PVAc and 0.5-2 wt\% magnetic fluid in DCM (Fig. 2a). These polymer concentrations allowed to fully dissolve both polymers in the organic phase and to achieve their complete segregation after DCM evaporation. Magnetic PS particles were prepared from the dispersed phase containing 4 wt $\%$ PS and 0.5-4 wt\% magnetic fluid in DCM (Fig. 2b). The continuous phase was a mixture of $2 \mathrm{wt} \%$ PVA and $30 \mathrm{wt} \%$ glycerol in DI, optimised to prevent droplet coalescence and achieve efficient flow focusing and preparation of monodispersed droplets at the investigated fluid flow rates. The polymer concentrations in the dispersed phase were selected to achieve a complete polymer phase separation after DCM evaporation without any patchy regions on the surface. ${ }^{23}$ Janus particles are formed from patchy particles through the patch coarsening process via Oswald ripening. During this process, the polymer diffuses away from smaller patches and the dissolved polymer is deposited onto larger patches to reduce the overall interfacial free energy of the system. As a result, larger patches grow at the expense of smaller ones until all patches disappear and the two polymers become fully separated. At high polymer concentrations in the dispersed phase, the Ostwald ripening is supressed due to lower diffusivity of polymer chains and it becomes more difficult for the polymers to fully separate from each other.

The prepared emulsions were collected in a vial pre-filled with the continuous phase solution to minimise contacts between the droplets and increase the rate of solvent removal. The solvent was evaporated at room temperature. After complete evaporation of DCM, the particles were washed with Milli-Q water several times and dried for further characterisation. 

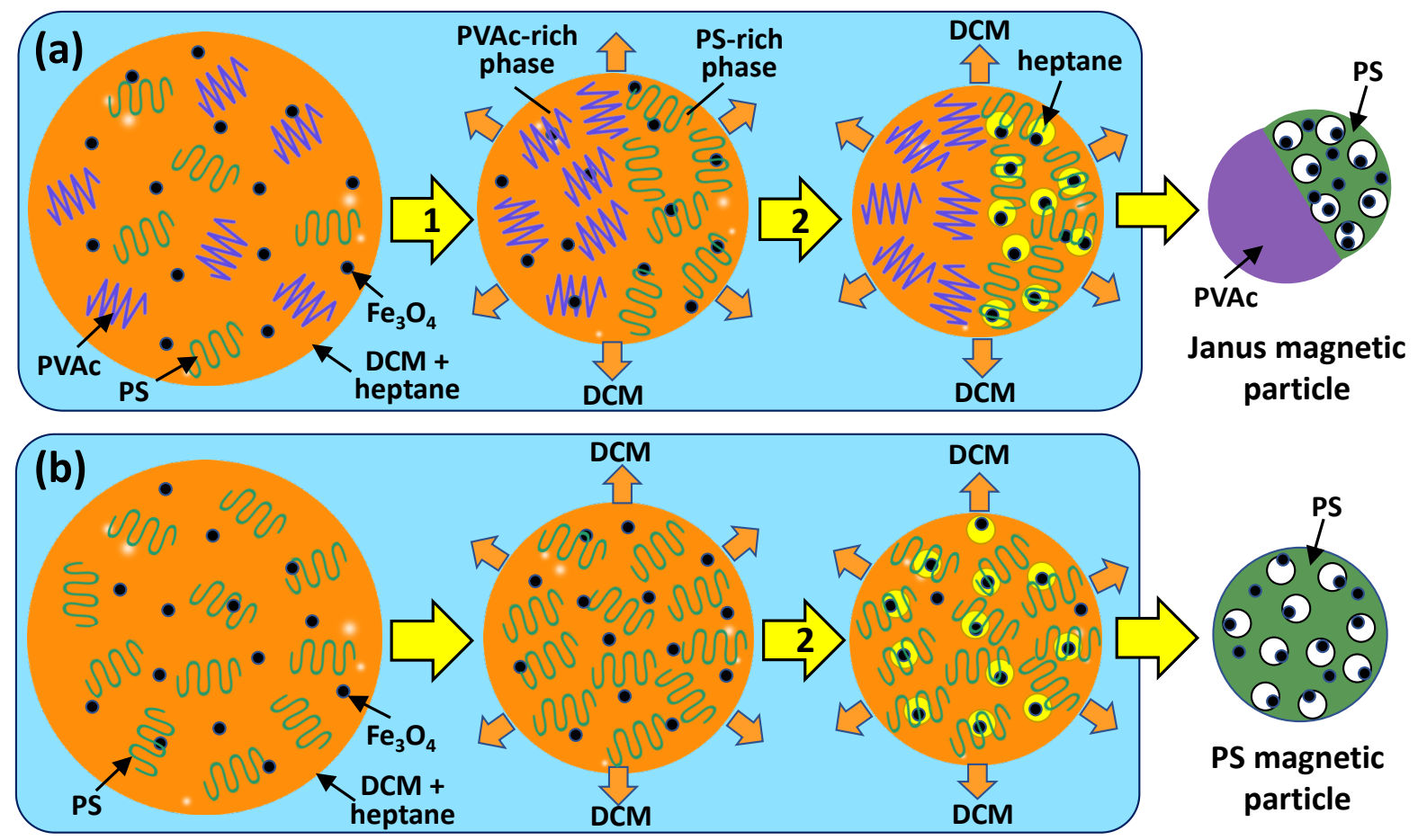

DCM $00 \Omega$ PS MW PVAC water $\bullet \mathrm{Fe}_{3} \mathrm{O}_{4} \bigcirc n$-heptane $\bigcirc$ pore

Fig 2. Schematic view of the formation of magnetic polymeric particles in this study: (a) Janus PS/PVAc particles with anisotropic (asymmetric) porosity and magnetic properties; (b) PS particles with isotropic (symmetric) porosity and magnetic properties. DCM - Dichloromethane; PS - Polystyrene; PVAc - Poly(vinyl acetate), $\mathrm{Fe}_{3} \mathrm{O}_{4}$ - Oleic acid-capped magnetic nanoparticles, 1: Phase separation between PS and PVAc, 2: Phase separation between DCM and $n$-heptane.

\section{Particle characterisation}

Optical microscopy. The JPs were observed with an optical microscope GXM L3201 (GT Vision Ltd) and RETIGA 6000 color CCD camera (QImaging, Canada) interfaced to a PC computer with Q-Capture Pro V.7.0 software.

Confocal Laser Scanning Microscopy (CLSM). The JPs stained with Nile Red were observed using a CLSM system (BIO-RAD Radiance2000 MP) mounted on a Nikon Eclipse TE300 microscope connected to a computer running Zeiss LaserSharp 2000 software. The sample was excited with an argon laser (14 $\mathrm{mW}$ at $488 \mathrm{~nm})$ and a helium-neon laser (1.5 mW at $543 \mathrm{~nm})$. A $570 \mathrm{~nm}$ long-pass emission filter was used for image acquisition via confocal microscopy.

\section{Scanning Electron Microscopy (SEM)-Energy Dispersive X-Ray Spectroscopy (SEM-EDX).}

The morphology and elemental composition of the particles were analysed using a TM3030 benchtop SEM (Hitachi, Tabletop Microscope Europe) operating at an accelerating voltage of 15 
$\mathrm{keV}$. The particles were coated with a $80: 20$ gold/palladium alloy prior to SEM to prevent accumulation of electrostatic charges on the polymer surface. The sputtering rate was $0.85 \mathrm{~nm} / \mathrm{s}$ at $2 \mathrm{kV}$ and $25 \mathrm{~mA}$ of plasma current.

Focused Ion Beam (FIB) Imaging. This imaging was carried out using a dual-beam focused ion beam scanning electron microscope (FIB-SEM) (Nova 600 Nanolab, FEI Company, Hillsboro, Oregon, USA).

Differential Scanning Calorimetry (DSC). Modulated DSC analysis was done using a TA Instruments model 2910 calorimeter. $10-15 \mathrm{mg}$ of the sample was placed in an aluminum pan and hermetically sealed. The sample pan was heated from 200 to $420^{\circ} \mathrm{C}$ at a rate of $1.5^{\circ} \mathrm{C} / \mathrm{min}$ and the difference in heat flow rate between the sample and empty pan was measured. The purge gas flow rate was maintained at $60 \mathrm{~mL} / \mathrm{min}$.

Attenuated total reflection Fourier transform infrared (ATR-FTIR) spectroscopy. ATRFTIR spectra in the range of 4000-400 cm-1 were recorded on a Thermo Scientific Nicolet iS50 ATR spectrometer with a monolithic diamond crystal. 2-3 mg of the sample was placed onto the Universal diamond ATR top-plate and the spectrum was acquired within $32 \mathrm{~s}$.

\section{Magnetic manipulation of particles}

Neodymium (NdFeB) magnets (grade N42, Aitsite Magnet Expert, UK) were used to test the responsiveness of the prepared particles to an external magnetic field. The particles were suspended in a Petri dish and manipulated by two magnets placed on each side of the Petri dish at the distance of $2 \mathrm{~cm}$.

\section{RESULTS AND DISCUSSION}

\section{Formation of Janus particles}

A polymer is soluble in a solvent if the Hansen solubility parameters for the solvent lie within the solubility sphere for the polymer, ${ }^{49-50}$ i.e. if $D_{S-P}<R_{0}$, where $R_{0}$ is the radius of the solubility curve and $D_{S-P}$ the distance of the solvent from the center of the polymer solubility sphere given by:

$$
D_{S-P}=\sqrt{4\left(\delta_{d, S}-\delta_{d, P}\right)^{2}+\left(\delta_{p, S}-\delta_{p, P}\right)^{2}+\left(\delta_{h, S}-\delta_{h, P}\right)^{2}}
$$


where $\delta_{d}, \delta_{p}$, and $\delta_{h}$ are the dispersion, polar, and hydrogen bonding components of the Hansen solubility parameter, and subscripts $\mathrm{S}$ and $\mathrm{P}$ refer to the solvent and polymer respectively. The values of the solubility parameters of the main components are shown in Table 1.

Table 1. The dispersion, $\delta_{d}$, polar, $\delta_{p}$, and hydrogen bonding $\delta_{h}$ component of the Hansen solubility parameters for polystyrene (PS), poly(vinyl acetate) (PVAc), dichloromethane (DCM), $n$-heptane (Hp), oleic acid, and water, and the radius of the solubility curve (the polymer interaction radius), $R_{0}$ for PS and PVAc.

\begin{tabular}{|c|c|c|c|c|}
\hline & $\delta_{d}\left(\mathrm{MPa}^{1 / 2}\right)$ & $\delta_{p}\left(\mathrm{MPa}^{1 / 2}\right)$ & $\delta_{h}\left(\mathrm{MPa}^{1 / 2}\right)$ & $R_{0}\left(\mathrm{MPa}^{1 / 2}\right)$ \\
\hline Polystyrene $^{51}$ & 21.3 & 5.8 & 4.3 & 12.7 \\
\hline Poly(vinyl acetate $)^{51}$ & 20.9 & 11.3 & 9.6 & 13.7 \\
\hline Dichloromethane $e^{51}$ & 18.2 & 6.3 & 6.1 & - \\
\hline n-heptane $\mathrm{e}^{51}$ & 15.3 & 0 & 0 & - \\
\hline Oleic acid ${ }^{52}$ & 16.5 & 3.1 & 5.7 & - \\
\hline Water $^{51}$ & 15.6 & 16 & 42.3 & - \\
\hline
\end{tabular}

The radius of the solubility sphere, $R_{0}$ has a value of $12.7 \mathrm{MPa}^{1 / 2}$ and $13.7 \mathrm{MPa}^{1 / 2}$ for PS and PVAc respectively. Applying Eq. (1) to the data in Table 1 gives $D_{D C M-P S}=6.5, D_{D C M-P V A_{C}}=8.1$, $D_{H p-P S}=14.0$, and $D_{H p-P V A c}=18.6\left(\right.$ all in $\left.\mathrm{MPa}^{1 / 2}\right)$. Therefore, DCM can be regarded as a good solvent for both polymers (because $D_{D C M-P S}<12.7 \mathrm{MPa}^{1 / 2}$ and $D_{D C M-P V A_{C}}<13.7 \mathrm{MPa}^{1 / 2}$ ), while $n$-heptane is a poor solvent for both polymers. PVAc is more polar than PS (because $\delta_{p, P V A_{c}}>$ $\left.\delta_{p, P S}\right)$, due to the presence of ester groups in vinyl acetate units and forms stronger hydrogen bonds than PS, since $\delta_{h, P V A_{c}}>\delta_{h, P S}$. On the other hand, $n$-heptane molecules have no functional groups (apart from $-\mathrm{CH}_{2}$ and $\left.-\mathrm{CH}_{3}\right)$ and interact only through dispersion forces $\left(\delta_{p, H p}=0, \delta_{h, H p}=0\right)$. Therefore, $n$-heptane shows a greater affinity to less polar PS than to PVAc and was accumulated in PS rich regions after phase separation. Oleic acid molecule has a similar $\delta_{d}$ value as $n$-heptane due to its long hydrocarbon chain. However, it is also slightly polar due to the presence of carboxylic head group. The negatively charged carboxylic groups of oleic acid molecules are bound to the positively charged surface of iron oxide nanoparticles while nonpolar hydrocarbon chains are exposed to $n$-heptane and thus, oleic-acid coated iron oxide nanoparticles should have a strong affinity to $n$-heptane and be concentrated in $n$-heptane rich regions. 
Freshly produced emulsion droplets were pipetted into a drop of the continuous phase placed on microscope slide and a series of time-lapse microscopic images were captured at regular intervals (Fig. 3 and Movie 1). The produced droplets were highly monodispersed with the size variations of less than 3\%. Obviously, the droplets at the edge of the lattice in Figs. 3 (a) and (b) were smaller than those away from the edge, because they were near the air interface and DCM was removed faster from these droplets. The volume of the smallest Janus particles in Fig. 3(b) was 10\% of the initial droplet volume. Since the volume percent of non-volatile species in the dispersed phase was $5.6 \%$, DCM was not fully removed from the particles, due to insufficient evaporation time. The PS portions appeared darker on optical images due to higher concentration of embedded iron oxide nanoparticles and absorption of light within their porous matrix. In addition, the PS parts occupied a larger volume fraction of the particles, although the mass fraction of both polymers was the same. It can be explained by the presence of residual DCM and $n$-heptane mainly in PS compartments and a smaller density of PS polymer compared to PVAc.

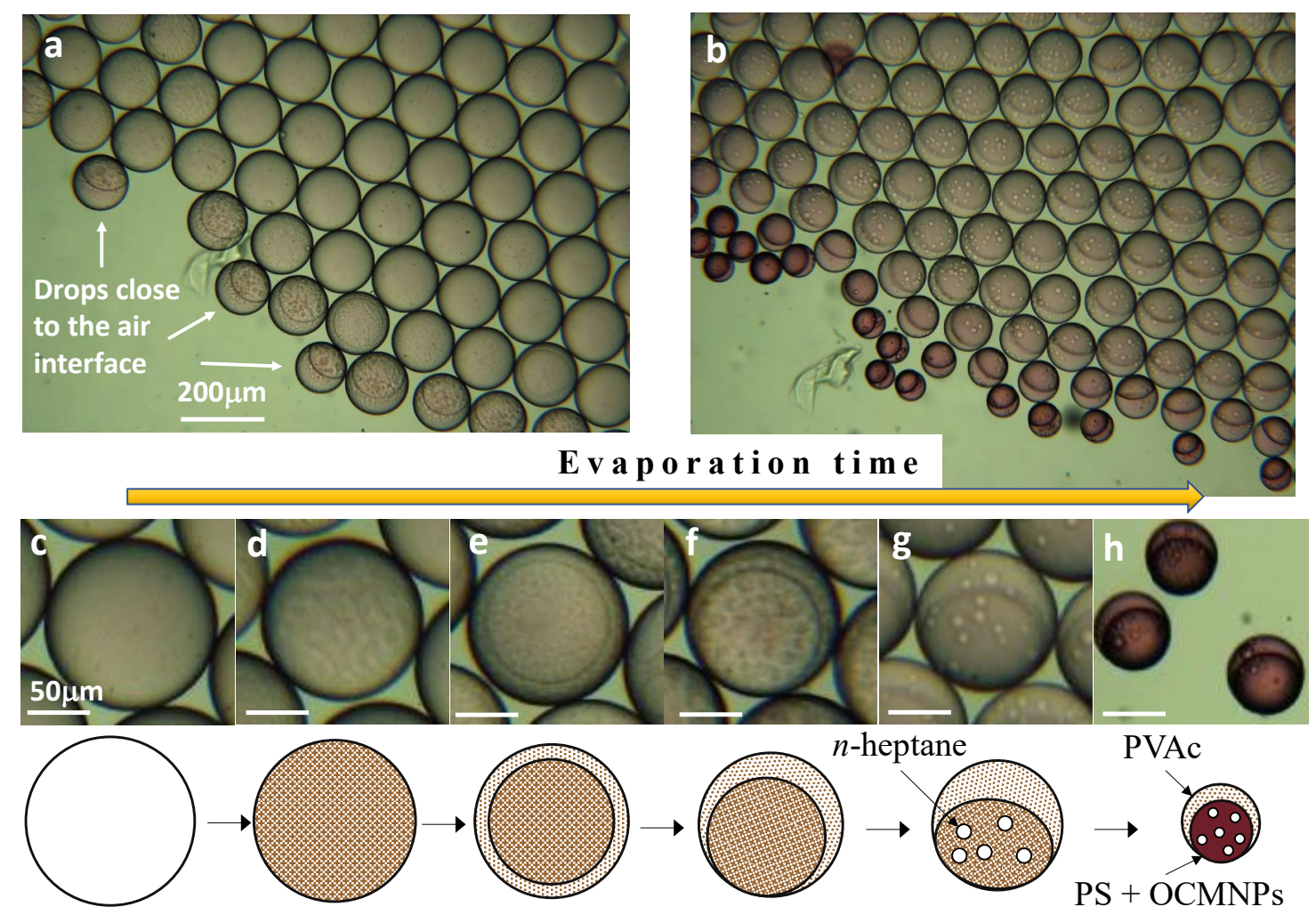

Fig 3. The progression of phase separation within droplets on a microscope slide: (a) Freshly prepared sample; (b) Phase-separated droplets/particles. (c-h) The transformation of a homogenous droplet into Janus particle due to DCM evaporation. The dispersed phase was $2 \mathrm{wt} \% \mathrm{PS}+2 \mathrm{wt} \%$ 
PVAc $+0.5 \mathrm{wt} \%$ magnetic fluid in DCM. The initial droplet diameter was $d_{\text {drop }}=135 \mu \mathrm{m}$ and the final particle diameter was $63 \mu \mathrm{m}$. The theoretical particle diameter based on a complete DCM evaporation is given by: $d_{\text {particle }}=d_{d r o p} \sqrt[3]{0.0564}=52 \mu \mathrm{m}$, where 0.0564 is the volume fraction of nonvolatile species in the dispersed phase.

Both polymers were initially fully miscible in the dispersed phase due to small concentration of $n$ heptane and therefore, the original droplet was transparent (Fig. 3c). As DCM diffused away, the concentration of $n$-heptane and both polymers increased within the droplets and eventually, the polymer solubility limit was reached, first for more polar PVAc and then for PS. The polymer precipitation started when the droplet volume was reduced by $13 \%$ and resulted in a thin solid skin on the droplet surface (Fig. 3d), which evolved into a core-shell structure (Fig. 3e). A PVAc-rich shell was exposed to the aqueous phase and a core containing more hydrophobic components (PS, $n$-heptane and DCM) was isolated from the aqueous phase by the shell. Fig. 3(f) shows the "acorn" morphology occurred when the initial droplet volume was reduced by $52 \%$. The acorn morphology is favored when $S_{P S}<0, S_{P V A C}<0$, and $S_{W}<0$, where $S_{P S}, S_{P V A C}$, and $S_{W}$ are the spreading coefficients for PS, PVAc, and aqueous phase respectively:

$$
\begin{aligned}
& S_{P S}=\sigma_{P V A C, W}-\left(\sigma_{P S, P V A c}+\sigma_{P S, W}\right) \\
& S_{P V A C}=\sigma_{P S, W}-\left(\sigma_{P S, P V A C}+\sigma_{P V A c, W}\right) \\
& S_{W}=\sigma_{P S, P V A c}-\left(\sigma_{P S, W}+\sigma_{P V A c, W}\right)
\end{aligned}
$$

where $\sigma_{P S, W}$ is the surface energy between PS and the aqueous phase, $\sigma_{P V A c, W}$ is the surface energy between PVAc and the aqueous phase, and $\sigma_{P S, P V A C}$ is the surface energy between PS and PVAc. When $S_{P S}<0, S_{P V A C}<0$, and $S_{W}<0$, the particles adopt a core/shell morphology with a PS core and PVAc shell. Our observation that PS and PVAc form composite particles with the acorn morphology was confirmed previously. ${ }^{33}$ Probably, core-shell morphology is a non-equilibrium transitional morphology created due to fast DCM evaporation from the interfacial region of the droplets. Fig. 3(g) shows droplets of $n$-heptane formed within phase-separated droplets upon further DCM evaporation. The point at which the phase separation between polymer-rich solvent and polymer-depleted non-solvent occurs depends upon the nature of the solvent and non-solvent 
and their compatibility with the polymer matrix. The formed $n$-heptane droplets with solubilised magnetic nanoparticles migrated into PS regions due to higher affinity of apolar $n$-heptane towards PS and acted as templates for the pore formation.

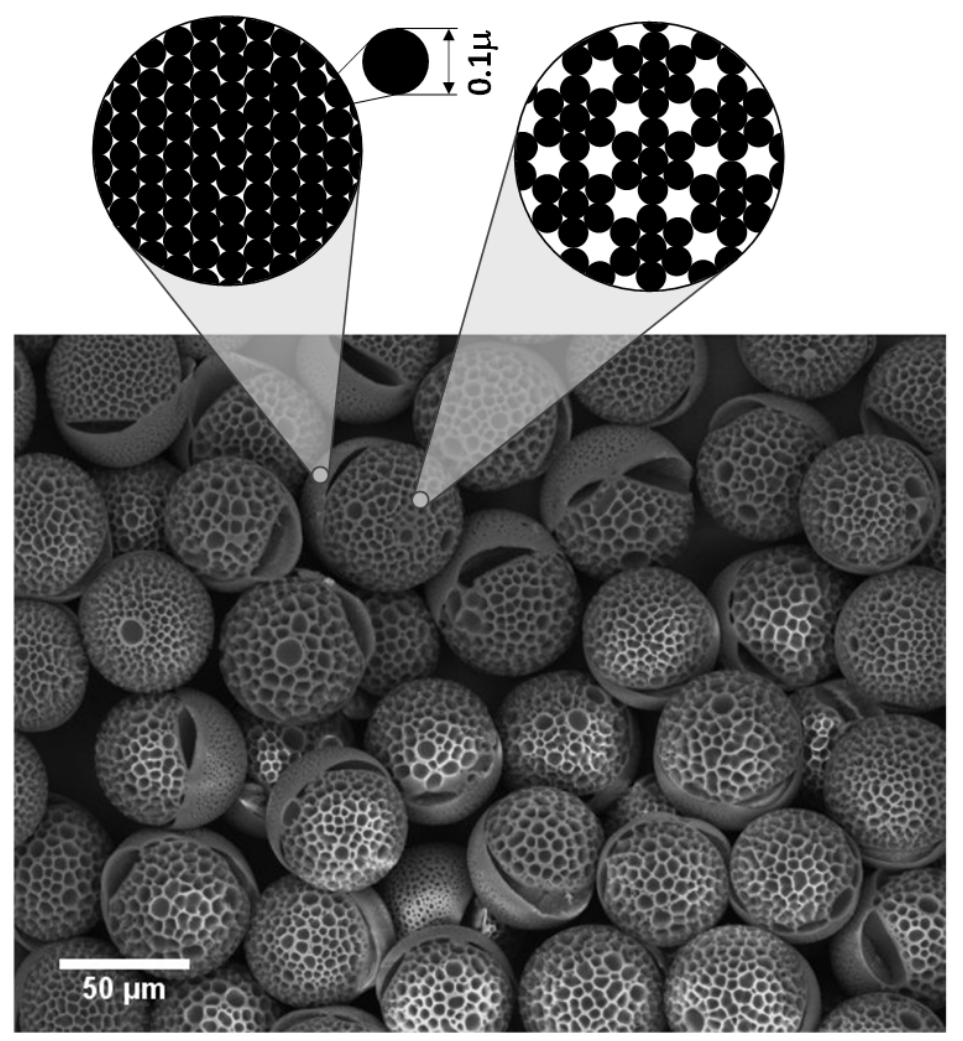

Fig 4. SEM image of monodispersed magnetic Janus particles with a diameter of $54 \mu \mathrm{m}$. Close-up images show primary microparticles (roughly $0.1 \mu \mathrm{m}$ in diameter) within polymer matrices of porous PS regions and non-porous PVAc regions. The dispersed phase was $2 \mathrm{wt} \% \mathrm{PS}+2 \mathrm{wt} \%$ PVAc $+1.5 \mathrm{wt} \%$ magnetic fluid in DCM.

As DCM evaporates, the polymer precipitates within the droplets and forms insoluble nuclei, which grow into spherical "microgel particles" with a typical diameter of $0.1 \mu \mathrm{m} .{ }^{53}$ In the absence of porogen, microgel particles aggregate within droplets and form a non-porous matrix composed of densely packed interconnected microgel particles. This mechanism occurs within a PVAc-rich phase due to small amount of $n$-heptane. A porous matrix is formed when a poor solvent is present in the polymer solution in addition to a good solvent, for example within a PS-rich phase, which causes phase separation of the polymer solution into discrete polymer-depleted non-solvent regions dispersed in a polymer-rich solvent phase. Due to low adhesion forces between the two 
polymers, Janus particles tend to form large cracks (mouth-like voids) between the two polymer hemispheres after drying, as shown in Fig. 4. These large cracks represent additional morphological feature of the prepared particles and can be exploited to entrap large entities, such as cells, within the Janus particles. The formation of cracks can be minimised by reducing the amount of nonsolvent added to the dispersed phase, leading to more compact PS matrix with smaller porosity. The morphology of our JPs was clearly different to that of PS/PVAc JPs prepared by Min et al. ${ }^{33}$ without adding any non-solvent to the dispersed phase. Their JPs exhibited different surface roughness of PS and PVAc parts, but both parts were non-porous.

\section{Particle characterisation}

\section{ATR-FTIR and DSC}

The chemical composition of the Janus particles was characterised using ATR-FTIR. The presence of PS was confirmed by the aromatic C-C stretching peaks at $1400-1600 \mathrm{~cm}^{-1}(\bullet)$ and the out-ofplane $=\mathrm{C}-\mathrm{H}$ bending at $690 \mathrm{~cm}^{-1}(\boldsymbol{\bullet})$ corresponding to the styrene aromatic rings in PS chains (Fig. 5a). The presence of PVAc was confirmed by the $\mathrm{C}=\mathrm{O}$ peak at $1730 \mathrm{~cm}^{-1}(\star)$ and the $\mathrm{C}-\mathrm{O}$ peak at $1200 \mathrm{~cm}^{-1}(\mathbf{\Delta})$ from ester groups. All main peaks from both polymers $(\boldsymbol{\bullet}, \bullet$, $\star$, and $\mathbf{\Delta})$ were present in the spectrum of the Janus particles, confirming that these particles were composed of two segregated polymers. Apart from the main peaks marked in Fig. 5(a), the aromatic $=\mathrm{C}-\mathrm{H}$ stretching peak was observed at $3100-3000 \mathrm{~cm}^{-1}$ in the spectrum of PS in addition to the alkane C$\mathrm{H}$ stretching peak slightly below $3000 \mathrm{~cm}^{-1}$, which was also observed in the PVAc spectrum.

Fig. 5(b) shows the thermal transitions of the produced Janus particles and individual polymers determined through DSC measurements. The DSC thermogram of PS shows one peak at $107^{\circ} \mathrm{C}$ corresponding to the glass transition temperature, $\mathrm{T}_{\mathrm{g}}$ of PS, while the DSC thermogram of PVAc shows two peaks at $32{ }^{\circ} \mathrm{C}$ and $52{ }^{\circ} \mathrm{C}$ corresponding to the glass transition temperature and the melting point of PVAc, respectively. The DSC thermogram of the Janus particles exhibits 3 peaks corresponding to the phase transition temperatures of both polymers indicating that the two polymers were not homogeneously distributed within the particles. 
(b)
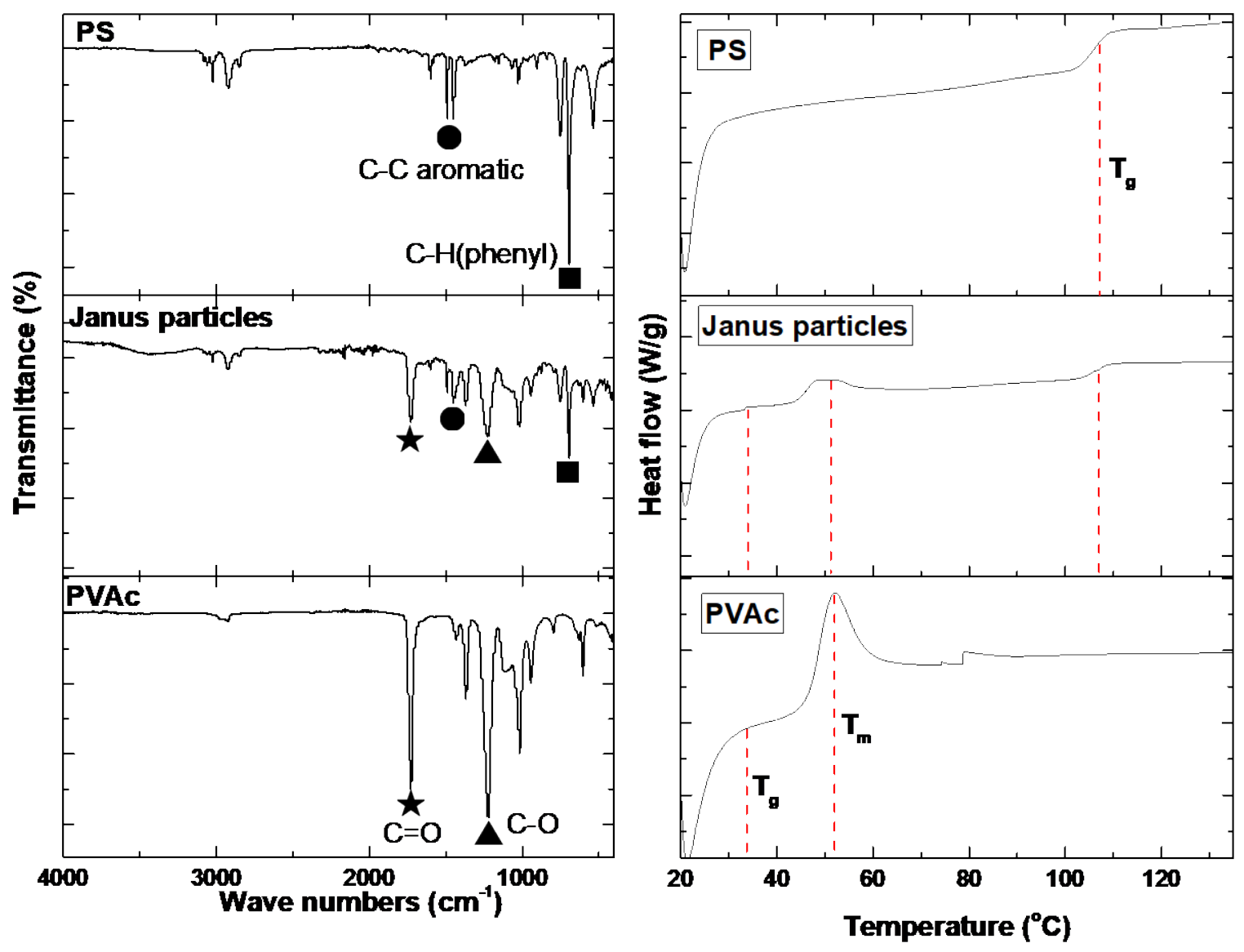

Fig 5. (a) ATR-FTIR spectra correlating transmittance vs. wave number for PS only, PVAc only, and Janus particles; (b) DSC thermal profiles for PS only, PVAc only, and Janus particles.

\section{SEM}

SEM images of the prepared magnetic polymer particles are shown in Fig. 6. Magnetic PS particles with negligible porosity were formed when $0.5 \mathrm{wt} \%$ of the magnetic fluid was added to the dispersed phase containing $4 \mathrm{wt} \%$ PS in DCM (Fig. 6a). When the magnetic fluid content in the dispersed phase was increased to $4 \mathrm{wt} \%$, highly porous magnetic PS particles were formed (Fig. $6 \mathrm{~b})$. This finding is consistent with a previous research indicating that the porosity of polymer particles generated by solvent evaporation can be tailored by adjusting the amount of non-solvent in the dispersed phase composed of a mixture of polymer, solvent and non-solvent. ${ }^{54}$ When a highly volatile non-solvent (2-methylpentane) was used, it was quickly removed from the surface 
region of the particles and the particles had a non-porous dimpled surface with a porous interior. ${ }^{55-56}$ However, $n$-heptane is much less volatile than 2-methylpentane, which resulted in a porous particle surface in this work. Porous particles shown in Fig. 6(b) can be used in various applications requiring high surface area per unit volume including sorption, chromatography, catalytic reactions, and ion exchange.

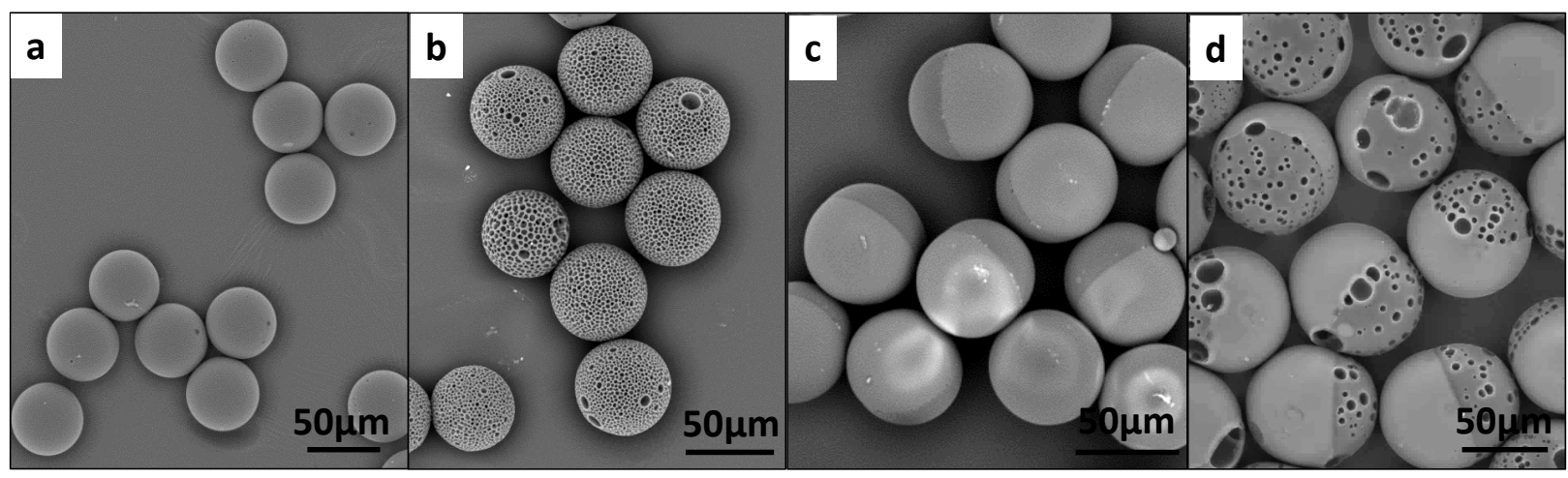

Fig 6. SEM images of the produced particles: (a) Non-porous magnetic PS particle, the dispersed phase: $4 \mathrm{wt} \%$ PS $+0.5 \mathrm{wt} \%$ magnetic fluid in DCM; (b) Porous magnetic PS particles, the dispersed phase: $4 \mathrm{wt} \%$ PS $+4 \mathrm{wt} \%$ magnetic fluid in DCM (see also Fig. 2b); (c) Non-porous Janus magnetic PS/PVAc particles, the dispersed phase: $2 \mathrm{wt} \% \mathrm{PS}+2 \mathrm{wt} \% \mathrm{PVAc}+0.5 \mathrm{wt} \%$ magnetic fluid in DCM; (d) Porous Janus magnetic PS/PVAc particles, the dispersed phase: $2 \mathrm{wt} \%$ PS $+2 w t \%$ PVAc $+1 w t \%$ magnetic fluid in DCM (see also Fig. 2a).

Nonporous magnetic Janus particles were created when $0.5 \mathrm{wt} \%$ of the magnetic fluid was added to the dispersed phase containing $2 \mathrm{wt} \%$ PS and $2 \mathrm{wt} \%$ PVAc (Fig. 6c). It can be explained by the absence of solvent-nonsolvent phase separation due to small fraction of $n$-hexane in DCM.

When higher amount of magnetic fluid was added to the dispersed phase ( $1 \mathrm{wt} \%)$, Janus particles with asymmetric surface topology were formed (Fig. 6d). The introduction of small structural features on one side of the Janus particle is a topic of great interest. ${ }^{27}$ Surface pores created on one side of the particles could provide ideal nucleation sites for bubble generation in bubble-driven Janus micromotors propelled by a gas generating enzymatic reaction. Bubbles cannot easily be formed on a smooth spherical surface ${ }^{27}$ and the pore cavities can provide space for enzyme loading and additional surface area for enzyme attachment. Furthermore, surface roughness can enhance the hydrophobicity of polymers, which can be used to produce JPs with superhydrophobic regions. Janus particles composed of superhydrophobic and hydrophilic compartments can be used as superhydrophobic barriers for aqueous solutions. ${ }^{57}$ 
In Fig. 6d, the pores are uniform in size over the whole surface of PS parts except at the contact line between the two polymers and water, where some large pores are also present. Probably, the interfacial tension at the PVAc/PS/water contact line was higher than on the remaining part of the particle surface, which promoted migration of heptane droplets to that contact line in order to reduce the total surface energy of the system. Some heptane droplets coalesced at the polymerpolymer contact line due to their high local concentration and these merged droplets created large pores after particle consolidation. To obtain more homogeneous pores, the amount of non-solvent added to the dispersed phase should be limited. It should lead to the reduced concentration of nonsolvent droplets during DCM evaporation and lower likelihood of their coalescence.

\section{SEM-EDX}

EDX analysis was used to determine the elemental composition of individual regions on the Janus particle and map out the distribution of elements from the imaged area. Fig. 7 shows X-ray maps for $\mathrm{C}$ and $\mathrm{O}$ within the selected particle. The distribution of $\mathrm{C}$ (red) and $\mathrm{O}$ (green) was asymmetric with the porous part containing more $\mathrm{C}$ (Fig. 7c) and the nonporous part containing more $\mathrm{O}$ (Fig. 7d). The molecular formula of PVAc is $\left(\mathrm{C}_{4} \mathrm{H}_{6} \mathrm{O}_{2}\right)_{n}$ and that of PS is $\left(\mathrm{C}_{8} \mathrm{H}_{8}\right)_{n}$ and thus, the nonporous region was rich in PVAc whilst the porous region contained more PS. The presence of O in both regions shows that the polymers were not fully separated within the two parts.

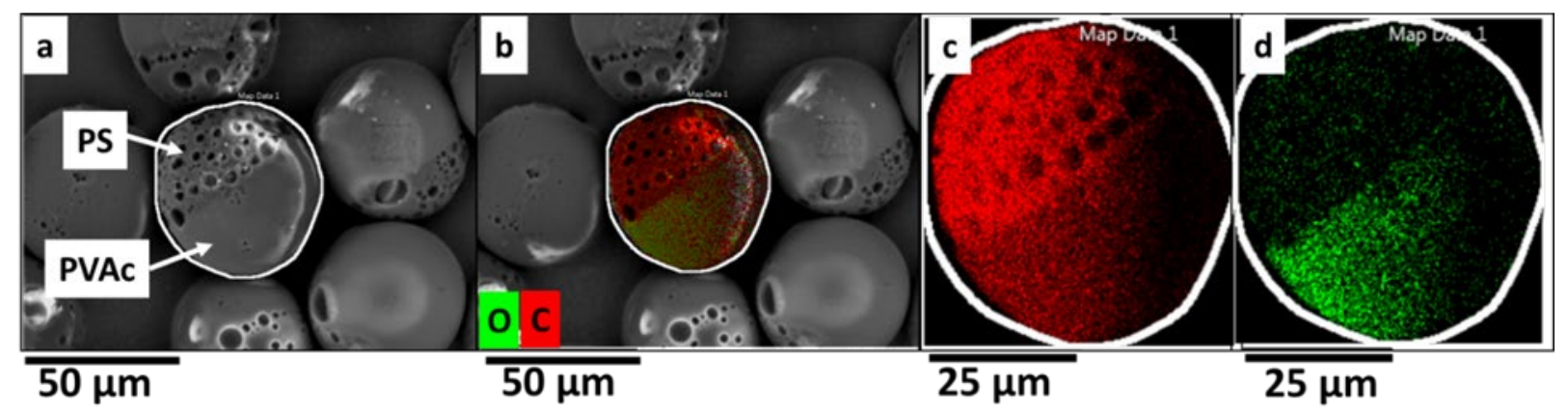

Fig 7. SEM-EDX elemental mapping for a Janus particle with a diameter of $50 \mu \mathrm{m}$ prepared from the dispersed phase composed of $2 \mathrm{wt} \% \mathrm{PS}, 2 \mathrm{wt} \%$ PVAc and $1 \mathrm{wt} \%$ magnetic fluid in DCM: (a) SEM image of the Janus particles, (b) Overlay X-ray map of carbon (C) and oxygen (O) with the SEM image superimposed; (c) The distribution map of $C$ in the particle; (d) The distribution map of $\mathrm{O}$ in the particle. The $\mathrm{C}$ (red) shows a higher intensity of X-ray in the porous PS compartment, as compared to the higher intensity of $\mathrm{O}$ (green) in the PVAc compartment.

Nanoparticles tend to migrate into the polymer phase which is more compatible with the coating material. Jeong et al. ${ }^{42}$ prepared magnetic Janus particles composed of poly(methyl methacrylate) 
(PMMA), poly(vinyl alcohol) (PVA), and poly(vinylpyrrolidone) (PVP) containing PVPstabilised magnetic nanoparticles. After solvent evaporation, the dispersed phase was separated into PMMA and PVP-PVA portions and the magnetic asymmetry of the particles was achieved due to higher affinity of PVP coating towards PVP-PVA parts. The novelty of our approach was in accumulation of magnetic nanoparticles into PS parts based on higher affinity of hydrocarbon chains of oleic acid coating towards PS hydrocarbon chains compared to polyvinyl ester chains of PVAc. We could not confirm asymetric distribution of Fe in the prepared Janus particles by EDX due to low sensitivity of the analysis. However, the accumulation of oleic acid-capped magnetic nanoparticles in PS regions was confirmed by the dark colour of PS domains on optical microscopy images, for example in Fig. 3(h).

\section{SEM-FIB and CLSM}

FIB was used for sectioning a single Janus particle and the exposed cross-sectional region was imaged using SEM (Fig. 8a). The particle was coated with platinum prior to FIB to protect the original surface from the high-energy ions during sectioning and imaging. The PVAc compartment is a crescent moon-shaped, reflecting the fact that PVAc is more hydrophilic and therefore tend to be more exposed to the aqueous phase during phase separation. The pores are located only at the surface region of the PS compartment, while the interior of the polymer matrix is non-porous.

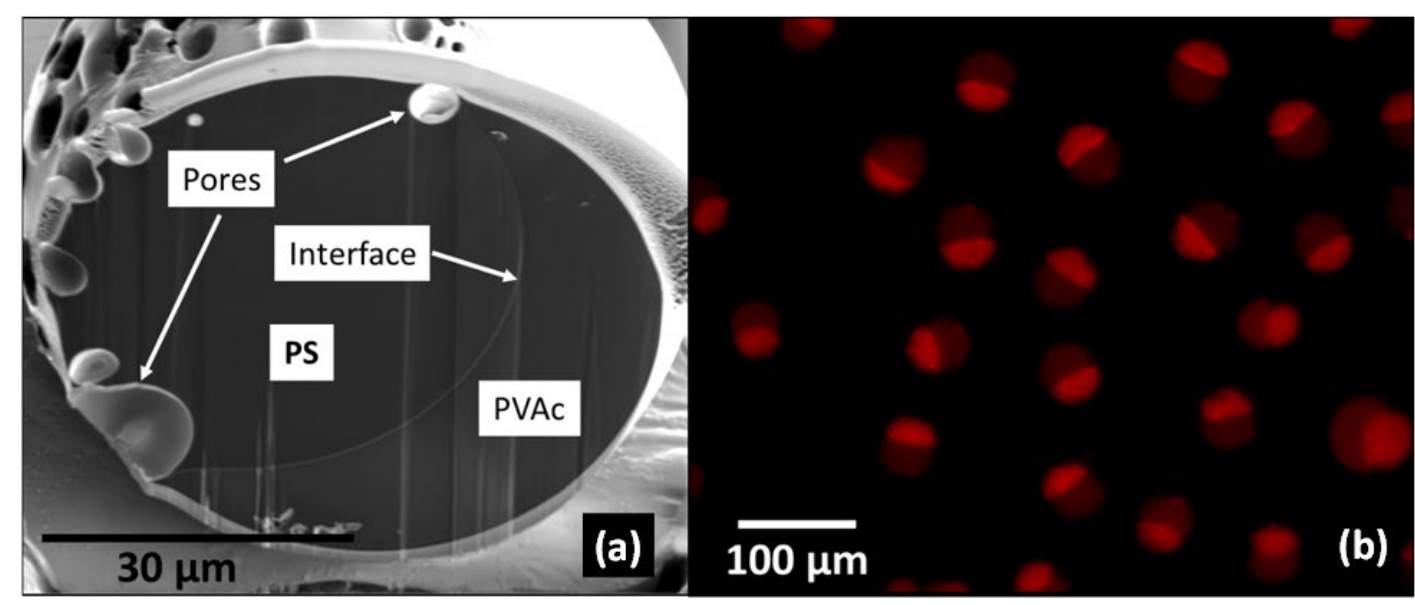

Fig 8. (a) FIB-SEM image of a single Janus particle; (b) CLSM image of the Janus particles stained with Nile Red. The dispersed phase: $2 \mathrm{wt} \% \mathrm{PS}+2 \mathrm{wt} \%$ PVAc $+1 \mathrm{wt} \%$ magnetic fluid in DCM.

Another method to distinguish between PS and PVAc compartments is to stain the particles with Nile Red, which is an uncharged hydrophobic molecule whose fluorescence is strongly influenced 
by the polarity of its environment. ${ }^{58}$ As shown in Fig. 8(b), Nile Red binds preferentially to apolar PS compartments. Preferential adsorption of dyes with opposite polarities can be used to generate bicolored particles, which can be introduced into honeycomb matrices and used as "electronic paper". ${ }^{59}$ If such pigments have thermochromic properties, they can even sense the environment temperature and change their color reversibly at specific temperatures. The combination of thermochromism and magnetism enables to create displays sensitive to both temperature and magnetic field. ${ }^{5}$ Site-specific adsorption can also be used to encapsulate two incompatible drugs with opposite polarities within the same particle if they need to be concurrently administrated at the controlled ratio.

\section{Magnetic manipulation of particles}

The fabricated particles were uniformly suspended in an aqueous PVA solution and then attracted by neodymium magnets. After short period of time, all particles were accumulated on a vertical cylindrical surface of the vial closest to the magnets, as shown in Fig. 9. Obviously, the gravitational force acting on the particles was counterbalanced with a magnetic force.

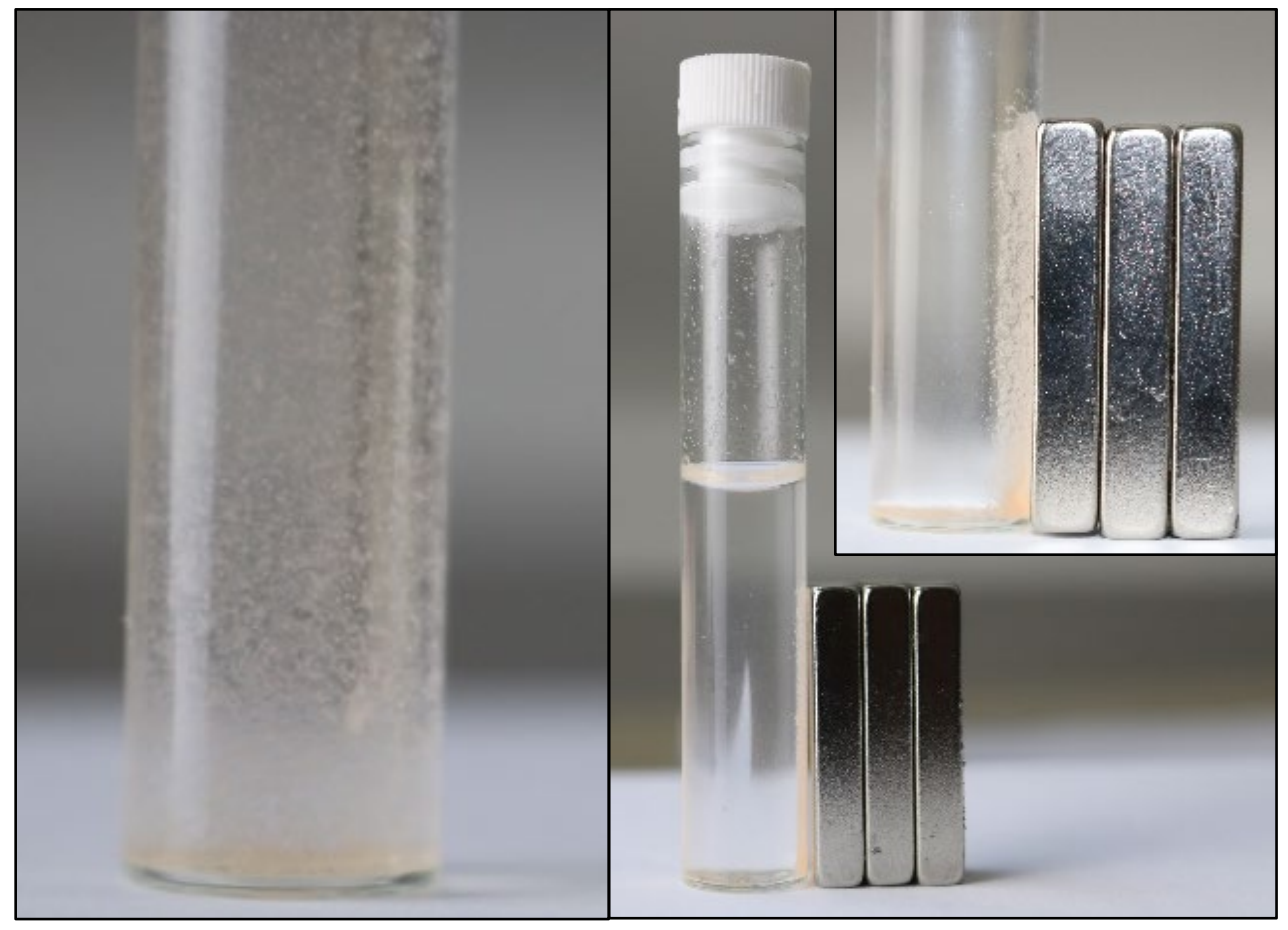

Fig 9. The attraction of the prepared Janus particles by strong stationary magnets (figure right and inset figure). Initially, the particles were uniformly dispersed in a PVA solution (figure left). 
After that, the fabricated particles suspended in a PVA solution were placed in a Petri dish and subjected to an external magnetic field generated by two neodymium magnets, which led to their rapid magnetisation and assembly into two-dimensional (2D) ordered structures. The process of magnetic-field-induced particle assembly was reversible and driven by temporary magnetic dipole-dipole interactions, which could be either attractive or repulsive, depending on the angle between the magnetic field and the line connecting the dipoles. ${ }^{60}$ Therefore, the particles could be quickly assembled into regular clusters and then disassembled when the magnetic field was removed. The size of the magnetic clusters depended on the strength of the applied magnetic field.

The particle self-assembly is one of the key bottom-up methods for the fabrication of functional smart materials. Magnetic clusters can be extracted from the dispersion medium and used in various application, for example in viscous dampers, for magnetic polishing, and for fabrication of composite materials such as magnetic rubber. ${ }^{61}$

Finally, when subjected to a rotating magnetic field, particle clusters composed of 2-4 individual particles were forced to rotate in the direction of magnetic field (Movie 2 and Fig. 10), which openup opportunities to use the particles as microrheological probes, active moving parts in constrained geometries or micro-stirrers. ${ }^{62}$ As shown in Fig. 10, magnetic nanoparticles were embedded only in PS parts of the particles imparting their dark colour. The asymmetric spatial distribution of $\mathrm{F}_{3} \mathrm{O}_{4}$ nanoparticles in polymer microparticles can also be achieved by applying the external magnetic field either during solvent evaporation ${ }^{63}$ or droplet generation in microfluidic channel. ${ }^{64}$ However, our facile method based on solvent-nonsolvent phase separation does not require the application of external magnetic field, with an added benefit that the porosity of the two particle segments can be selectively tuned.

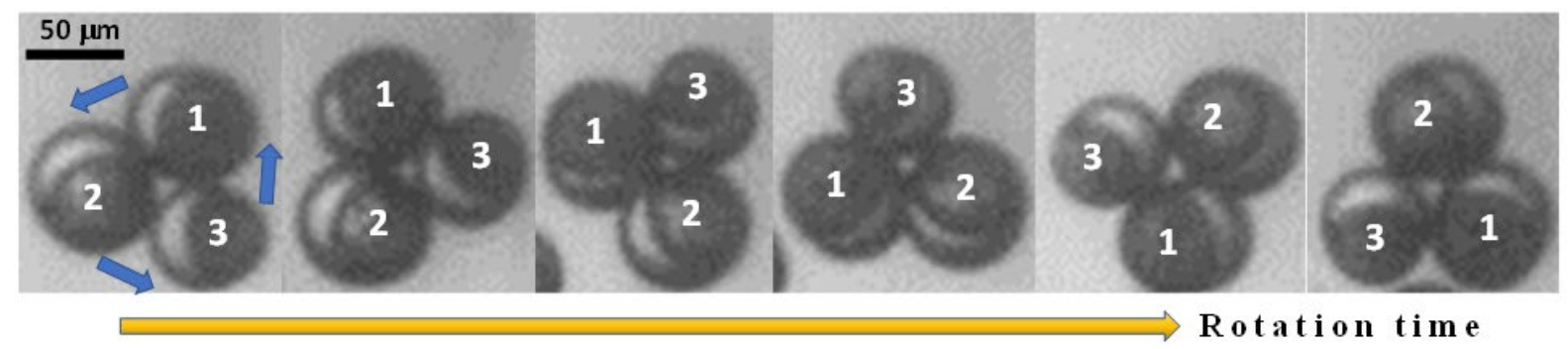

Fig 10. Optical microscopy images showing the collective rotational motion of a particle assembly consisted of three Janus particles under the external magnetic field. The dark compartments are 
porous PS regions loaded with magnetic nanoparticles, while transparent crescent-moon-shaped compartments are nonporous PVAc regions. The arrows represent the direction of motion of the cluster.

\section{CONCLUSIONS}

We have fabricated monodispersed magnetic polymer particles with both isotropic (symmetric) and anisotropic (asymmetric) properties. Anisotropic (Janus) particles with chemical, magnetic, and microstructural anisotropy were generated after two consecutive phase separation events in the quinary dispersed phase composed of two polymers (PVAc and PS), DCM, $n$-heptane, and oleic acid-coated magnetic nanoparticles. Isotropic particles with uniform properties over the entire volume have been prepared in the quaternary system composed of a single polymer (PS), DCM, $n$-heptane, and oleic acid coated magnetic nanoparticles. Anisotropic properties of the fabricated Janus particles were the result of the following phase separation phenomena within the template droplets: (a) PS-PVAc separation due to thermodynamic incompatibility between the two polymers, and (b) phase separation between a good solvent (DCM) and a poor solvent ( $n$-heptane) in PS parts, which led to high porosity of the PS matrix. Magnetic anisotropy of the prepared particles was achieved due to higher affinity of hydrocarbon tails of oleic acid molecules towards hydrocarbon polymer chains of PS compared to polyvinyl ester chains of PVAc. The accumulation of oleic acid capped magnetic nanoparticles in PS parts was confirmed by the dark appearance of PS regions on optical microscopy images.

The pore cavities in PS portions are a useful topological feature, which can be exploited to increase the rate of bubble generation in bubble-driven Janus micromotors, as well as to enhance waterrepellent properties of polystyrene portions. The asymmetric distribution of $\mathrm{C}$ and $\mathrm{O}$ within a Janus particle was confirmed by SEM-EDX. The pore size distribution in polystyrene parts was tailored by controlling the amount of magnetic fluid added to the dispersed phase. Bicoloured particles can be generated by preferential adsorption of pigments with opposite polarities onto hydrophilic and hydrophobic particle segments. If thermochromic pigments are used for polymer staining, the particles can change their colour in response to magnetic field. 
The microfluidic method developed in this work to prepare Janus particles can be extended to a variety of polymers and nanoparticles which may lead to the creation of versatile field-responsive Janus particles with various interesting topological features from fine pores to large.

\section{Supporting Information}

The following items are available free of charge:

- A time-lapse video of phase separation within the template droplets leading to the generation of Janus magnetic particles (movie 1).

- A video showing particle clustering in the external magnetic field due to their magnetization and the rotation of particle clusters in a rotating magnetic field whose direction was manually adjusted (movie 2).

\section{ACKNOWLEDGMENT}

The authors acknowledge a scholarship from the Government of Oman for Ruqaiya Al Nuumani, the support received from the EPSRC grant EP/HO29923/1, and the use of facilities within the Loughborough Materials Characterisation Centre (LMCC).

\section{REFERENCES}

(1) Kaewsaneha, C.; Tangboriboonrat, P.; Polpanich, D.; Eissa, M.; Elaissari, A. Janus Colloidal Particles: Preparation, Properties, and Biomedical Applications. ACS Appl. Mater. Interfaces 2013, 5, 1857-1869.

(2) $\mathrm{Du}$, J.; O'Reilly, R. K. Anisotropic Particles with Patchy, Multicompartment and Janus Architectures: Preparation and Application', Chem. Soc. Rev. 2011, 40, 2402-2416.

(3) Yin, S. N.; Wang, C. F.; Yu, Z. Y.; Wang, J.; Liu, S. S.; Chen, S. Versatile Bifunctional Magnetic-Fluorescent Responsive Janus Supraballs Towards the Flexible Bead Display. Adv. Mater. 2011, 23, 2915-2919.

(4) Liu, S. S.; Wang, C. F.; Wang, X. Q.; Zhang, J.; Tian, Y.; Yin, S. N.; Chen, S. Tunable Janus Colloidal Photonic Crystal Supraballs with Dual Photonic Band Gaps. J. Mater. Chem. C 2014, 2, 9431-9438.

(5) Cui, Y.; Wang, Y.; Wu, J.; He, X.; Xuan, S.; Gong, X. Magneto-Thermochromic Coupling Janus Sphere for Dual Response Display. RSC Adv. 2019, 9, 17959-17966. 
(6) Yi, Y.; Sanchez, L; Gao, Y.; Yu, Y. Janus Particles for Biological Imaging and Sensing. Analyst, 2016, 141, 3526-3539.

(7) Campuzano, S.; Gamella, M.; Serafín, V.; Pedrero, M.; Yáñez-Sedeño, P.; Pingarrón, H. M. Magnetic Janus Particles for Static and Dynamic (Bio)Sensing. Magnetochemistry 2019, 5, 47.

(8) Tu, F.; Lee, D. One-Step Encapsulation and Triggered Release Based on Janus ParticleStabilized Multiple Emulsions. Chem. Commun. 2014, 50, 15549-15552.

(9) Li, W.; Dong, H.; Tang, G.; Ma, T.; Cao, X. Controllable Microfluidic Fabrication of Janus and Microcapsule Particles for Drug Delivery Applications. RSC Adv. 2015, 5, 23181-23188.

(10)López, V.; Villegas, M. R.; Rodríguez , V.; Villaverde, G.; Lozano, D.; Baeza, A.; ValletRegí, M. Janus Mesoporous Silica Nanoparticles for Dual Targeting of Tumor Cells and Mitochondria. ACS Appl. Mater. Interfaces 2017, 9, 26697-26706.

(11)Behrend, C. J.; Anker, J. N.; McNaughton, B. H.; Brasuel, M.; Philbert, M. A.; Kopelman, R. Metal-Capped Brownian and Magnetically Modulated Optical Nanoprobes (Moons): Micromechanics in Chemical and Biological Microenvironments. J. Phys. Chem. B 2004, 108, 10408-10414.

(12) Ma, X.; Sanchez, S. A Bio-Catalytically Driven Janus Mesoporous Silica Cluster Motor with Magnetic Guidance. Chem. Commun. 2015, 51, 5467-5470.

(13) Wu, Z.; Li, L.; Liao, T.; Chen, X.; Jiang, W.; Luo, W.; Yang, J.; Sun, Z. Janus Nanoarchitectures: from Structural Design to Catalytic Applications. Nano Today 2018, 22, $62-82$.

(14)Xue, D.; Meng, Q. B.; Song, X. M. Magnetic-Responsive Janus Nanosheets with Catalytic Properties. ACS Appl. Mater. Interfaces 2019, 11, 10967-10974.

(15) Su, L.; Ma, J.; Wang, J.; Jiang, W.; Zhang, W.; Yang, J. Site-Selective Exposure of Iron Nanoparticles to Achieve Rapid Interface Enrichment for Heavy Metals. Chem. Commun. 2020, 56, 2795-2798.

(16) Walther, A.; Hoffmann, M.; Müller, A. H. E. Emulsion Polymerization Using Janus Particles as Stabilizers. Angew. Chem., Int. Ed. 2008, 47, 711-714.

(17) Tanaka, T.; Okayama, M.; Minami, H.; Okubo, M. Dual Stimuli-Responsive "MushroomLike” Janus Polymer Particles as Surfactants Particulate. Langmuir 2010, 26, 11732-11736.

(18)Wei, D.; Ge, L.; Lu, S.; Li, J.; Guo, R. Janus Particles Templated by Janus Emulsions and 
Application as a Pickering Emulsifier. Langmuir 2017, 33, 5819-5828.

(19) Jiang, S.; Chen, Q.; Tripathy, M.; Luijten, E.; Schweizer, K. S.; Granick, S. Janus Particle Synthesis and Assembly. Adv. Mater. 2010, 22, 1060-1071.

(20)Kirillova, A.; Marschelke, C.; Synytska, A. Hybrid Janus Particles: Challenges and Opportunities for the Design of Active Functional Interfaces and Surfaces. ACS Appl. Mater. Interfaces 2019, 11, 9643-9671.

(21)Huang, X.; Qian, Q.; Wang, Y. Anisotropic Particles From a One-Pot Double Emulsion Induced by Partial Wetting and Their Triggered Release. Small 2014, 10, 1412-1420.

(22) Winkler, J. S.; Barai, M.; Tomassone, M. S. Dual Drug-Loaded Biodegradable Janus Particles for Simultaneous Co-Delivery of Hydrophobic and Hydrophilic Compounds. Exp. Biol. Med. $2019,244,1162-1177$.

(23)Ekanem, E. E.; Zhang, Z.; Vladisavljević, G. T. Facile Production of Biodegradable Bipolymer Patchy and Patchy Janus Particles with Controlled Morphology by Microfluidic Routes. Langmuir 2017, 33, 8476-8482.

(24) Yi, Y.; Sanchez, L.; Gao, Y.; Lee, K.; Yu, Y. Interrogating Cellular Functions with Designer Janus Particles. Chem. Mater. 2017, 29, 1448-1460.

(25)Nie, Z.; Li, W.; Seo, M.; Xu, S.; Kumacheva, E. Janus and Ternary Particles Generated by Microfluidic Synthesis: Design, Synthesis, and Self-Assembly. J. Am. Chem. Soc. 2006, 128, 9408-9412.

(26) Ghosh, A.; Sheridon, N. K.; Fischer, P. Voltage-Controllable Magnetic Composite Based on Multifunctional Polyethylene Microparticles. Small, 2008, 4, 1956-1958.

(27)Kim, S. H.; Sim, J. Y.; Lim, J. M.; Yang, S. M. Magnetoresponsive Microparticles with Nanoscopic Surface Structures for Remote-Controlled Locomotion. Angew. Chem., Int. Ed. 2010, 49, 3786-3790.

(28) Tierno, P. Recent Advances in Anisotropic Magnetic Colloids: Realization, Assembly and Applications. Phys. Chem. Chem. Phys. 2014, 16, 23515-23528.

(29) McConnell, M. D.; Kraeutler, M. J.; Yang, S.; Composto, R. J. Patchy and Multiregion Janus Particles with Tunable Optical Properties. Nano Lett. 2010, 10, 603-609.

(30) Wolf, A.; Walther, A.; Müller, A. H. E. Janus Triad: Three Types of Nonspherical, Nanoscale Janus Particles from One Single Triblock Terpolymer. Macromolecules 2011, 44, 9221-9229.

(31) Bradley, L. C.; Stebe, K. J.; Lee, D. Clickable Janus Particles. J. Am. Chem. Soc. 2016, 138, 
11437-11440.

(32)Wang, Y.; Guo, B. H.; Wan, X.; Xu, J.; Wang, X.; Zhang, Y. P. Janus-Like Polymer Particles Prepared via Internal Phase Separation from Emulsified Polymer/Oil Droplets. Polymer, 2009, 50, 3361-3369.

(33) Min, N. G.; Choi, T. M.; Kim, S. H. Bicolored Janus Microparticles Created by Phase Separation in Emulsion Drops. Macromol. Chem. Phys. 2017, 218, 1600265.

(34) Yin, Y.; Lu, Y.; Gates, B.; Xia, Y. Template-Assisted Self-Assembly: A Practical Route to Complex Aggregates of Monodispersed Colloids with Well-Defined Sizes, Shapes, and Structures. J. Am. Chem. Soc. 2001, 123, 8718-8729.

(35) Perro, A.; Reculusa, S.; Ravaine, S.; Bourgeat-Lamic, E.; Duguet, E. Design and Synthesis of Janus Micro- and Nanoparticles. J. Mater. Chem. 2005, 15, 3745-3760.

(36) Shepherd, R. F.; Conrad, J. C.; Rhodes, S. K.; Link, D. R.; Marquez, M.; Weitz, D. A.; Lewis, J. A. Microfluidic Assembly of Homogeneous and Janus Colloid-Filled Hydrogel Granules. Langmuir 2006, 22, 8618-8622.

(37) Vladisavljević, G. T.; Al Nuumani, R; Nabavi, S. A. Microfluidic Production of Multiple Emulsions. Micromachines, 2017, 8, 1-34.

(38) Yuet, K. P.; Hwang, D. K.; Haghgooie, R.; Doyle, P. S. Multifunctional Superparamagnetic Janus Particles. Langmuir 2010, 26, 4281-4287.

(39) Yang, Y. T.; Wei, J.; Li, X.; Wu, L. J.; Chang, Z. Q.; Serra, C. A. A Side-By-Side CapillariesBased Microfluidic System for Synthesizing Size- and Morphology-Controlled Magnetic Anisotropy Janus Beads. Adv. Powder Technol. 2015, 26, 156-162.

(40) Nisisako, T.; Hatsuzawa, T. A Microfluidic Cross-Flowing Emulsion Generator for Producing Biphasic Droplets and Anisotropically Shaped Polymer Particles. Microfluid. Nanofluid. 2010, 9, 427-437.

(41) Walther, A.; Müller, A. H. E. Janus Particles: Synthesis, Self-Assembly, Physical Properties, and Applications. Chem. Rev. 2013, 113, 5194-5261.

(42) Jeong, J.; Um, E.; Park, J.K.; Kim, M. W. One-Step Preparation of Magnetic Janus Particles Using Controlled Phase Separation of Polymer Blends and Nanoparticles. RSC Adv. 2013, 3, 11801-11806.

(43)Cao, X.; Li, W.; Ma, T.; Dong, H. One-Step Fabrication of Polymeric Hybrid Particles with Core-Shell, Patchy, Patchy Janus and Janus Architectures via a Microfluidic-Assisted Phase 
Separation Process. RSC Adv. 2015, 5, 79969-79975.

(44)Biswal, A. K.; Saha, S. Controllable Fabrication of Biodegradable Janus and Multi-Layered Particles with Hierarchically Porous Structure. J. Colloid Interface Sci. 2020, 566, 120-134.

(45) Ge, L.; Cheng, J.; Sun, X.; Liu, J.; Wei, D.; Guo, R. Controlled Group Motion of Anisotropic Janus Droplets Prepared by One-Step Vortex Mixing. ACS Appl. Mater. Interfaces 2020, 12, $14588-14598$.

(46) Al Nuumani, R.; Bolognesi, G.; Vladisavljevic, G. T. Microfluidic Production of Poly (1,6Hexanediol Diacrylate)-Based Polymer Microspheres and Bifunctional Microcapsules with Embedded $\mathrm{TiO}_{2}$ Nanoparticles. Langmuir 2018, 34, 11822-11831.

(47) Bandulasena, M.; Vladisavljević, G. T.; Benyahia, B. Droplet-Based Microfluidic Method for Robust Preparation of Gold Nanoparticles in Axisymmetric Flow Focusing Device. Chem. Eng. Sci. 2019, 195, 657-664.

(48) Othman, R.; Vladisavljević, G. T.; Nagy, Z. K. Preparation of Biodegradable Polymeric Nanoparticles for Pharmaceutical Applications Using Glass Capillary Microfluidics. Chem. Eng. Sci. 2015, 137, 119-130.

(49)Hansen, C. M. Hansen Solubility Parameters: A User's Handbook - Second Edition. CRC Press, Boca Raton. 2007.

(50) Miller-Chou, B. A.; Koenig, J. L. A Review of Polymer Dissolution. Prog. Polym. Sci. 2003, $28,1223-1270$.

(51) Barton, A. F. M. CRC Handbook of Solubility Parameters and Other Cohesion Parameters, Second Edition, Taylor and Francis, London, 2017.

(52)De La Peña-Gil, A.; Toro-Vazquez, J. F.; Rogers, M. A. Simplifying Hansen Solubility Parameters for Complex Edible Fats and Oils. Food Biophys. 2016, 11, 283-291.

(53) Sherrington, D. C. Preparation, Structure and Morphology of Polymer Supports. Chem. Commun. 1998, 21, 2275-2286.

(54)Ekanem, E. E.; Nabavi, A. A.; Vladisavljević, G. T.; Gu, S. Structured Biodegradable Polymeric Microparticles for Drug Delivery Produced Using Flow Focusing Glass Microfluidic Devices. ACS Appl. Mater. Interfaces 2015, 7, 23132-23143.

(55) Hwangbo, K. H.; Kim, M. R.; Lee, C. S.; Cho, K. Y. Facile Fabrication of Uniform Golf-BallShaped Microparticles from Various Polymers. Soft Matter 2011, 7, 10874-10878.

(56) Kim, M. R.; Lee, S.; Park, J. K.; Cho, K. Y. Golf Ball-Shaped PLGA Microparticles with 
Internal Pores Fabricated by Simple O/W Emulsion. Chem. Commun. 2010, 46, 7433-7435.

(57)Kim, S. H.; Lee, S. Y.; Yang, S. M. Janus Microspheres for a Highly Flexible and Impregnable Water-Repelling Interface. Angew. Chem., Int. Ed. 2010, 49, 2535-2538.

(58) Sackett, D. L.; Wolff, J. Nile Red as a Polarity-Sensitive Fluorescent Probe of Hydrophobic Protein Surfaces. Anal. Biochem. 1987, 167, 228-234.

(59) Yabu, H. Colored Magnetic Janus Particles. IEICE Trans. Electron. 2017, E100.C, 955-957.

(60)Wang, M.; He, L.; Yin, Y. Magnetic Field Guided Colloidal Assembly. Mater. Today 2013, 16, 110-116.

(61) Shimada, K.; Shuchi, S.; Kanno, H.; Wu, Y.; Kamiyama, S. Magnetic Cluster and its Applications. J. Magn. Magn. Mater. 2005, 289, 9-12.

(62) Brasovs, A.; Cīmurs, J.; Ērglis, K.; Zeltins, A.; Berret, J. F.; Cēbers, A. Magnetic Microrods as a Tool for Microrheology. Soft Matter 2015, 11, 2563-2569.

(63) Chen, Y.; Nurumbetov, G.; Chen, R.; Ballard, N.; Bon, S.A.F. Multicompartmental Janus Microbeads from Branched Polymers by Single-Emulsion Droplet Microfluidics. Langmuir 2013, 29, 12657-12662.

(64) Varma, V.B.; Wu, R.G.; Wang, Z.P.; Ramanujan, R.V. Magnetic Janus Particles Synthesized using Droplet Micro-magnetofluidic Techniques for Protein Detection. Lab Chip 2017, 17, 3514-3525. 